\title{
Asteroid families interacting with secular resonances
}

\author{
V. Carruba ${ }^{\mathrm{a}, *}$, D. Vokrouhlický ${ }^{\mathrm{b}}, \mathrm{B}$. Novakovićc \\ a São Paulo State University (UNESP), School of Natural Sciences and Engineering, Guaratinguetá, SP, 12516-410, Brazil \\ ${ }^{\mathrm{b}}$ Institute of Astronomy, Charles University, $V$ Holešovičkách 2, Prague 8, CZ-18000, Czech Republic \\ ${ }^{\mathrm{c}}$ University of Belgrade, Faculty of Mathematics, Department of Astronomy, Studentski trg 16, 11000 Belgrade, Serbia
}

\section{A R T I C L E I N F O}

\section{Keywords:}

Minor planets

Asteroids: general

Celestial mechanics

\begin{abstract}
A B S T R A C T
Asteroid families are formed as the result of collisions. Large fragments are ejected with speeds of the order of the escape velocity from the parent body. After a family has been formed, the fragments' orbits evolve in the space of proper elements because of gravitational and non-gravitational perturbations, such as the Yarkovsky effect. Disentangling the contribution to the current orbital position of family members caused by the initial ejection velocity field and the subsequent orbital evolution is usually a difficult task. Among the more than 100 asteroid families currently known, some interact with linear and non-linear secular resonances. Linear secular resonances occur when there is a commensurability between the precession frequency of the longitude of the pericenter $(g)$ or of the longitude of node $(s)$ of an asteroid and a planet, or a massive asteroid. The linear secular resonance most effective in increasing an asteroid eccentricity is the $\nu_{6}$, that corresponds to a commensurability between the precession frequency $g$ of an asteroid and Saturn's $g_{6}$. Non-linear secular resonances involve commensurabilities of higher order, and can often be expressed as combinations of linear secular resonances. This is the case, for instance, of the $z_{k}=k\left(g-g_{6}\right)+\left(s-s_{6}\right)$ resonances. Asteroid families that are crossed by, or even have a large portion of their members, in secular resonances are of particular interest in dynamical astronomy. First, they often provide a clear evidence of asteroid orbit evolution due to the Yarkovsky effect. Second, conserved quantities of secular dynamics can be used to set valuable constraints on the magnitude of the original ejection velocity field. For the case of the $\nu_{6}$ secular resonance, objects in anti-aligned (paradoxal) librating states can be prevented to achieve high values of eccentricity and remain long-term stable (the case for members of the Tina family). Finally, by changing the value of inclination of family members, and, indirectly, of the $v_{W}$ component of the observed ejection velocity field, nodal secular resonances with massive asteroids or dwarf planets, such as the $s-s_{C}$ secular resonance with Ceres, can cause $v_{W}$ to become more and more leptokurtic (i.e., more peaked and with larger tails than that of a Gaussian distribution). By simulating fictitious asteroid families and by requiring that the current value of the Pearson kurtosis of $v_{W}, \gamma_{2}\left(v_{W}\right)$, be attained, independent constraints on the value of families ages can be obtained for families affected by these kinds of resonances.
\end{abstract}

\section{Introduction}

Among the more than 100 currently known asteroid families some are characterized by their interaction with linear or non-linear secular resonances. Asteroid families that are crossed, or even entirely immersed, in these resonances are of particular interest in dynamical astronomy. This is because they often provide evidence of long-term orbital evolution due to non-gravitational (Yarkovsky) forces and/or can be used to set independent constraints on the magnitude of the original ejection velocity field.

One issue that has to be first solved when dealing with asteroid families interacting with secular resonances is the orbital location of these resonances. (Hirayama, 1923) derived secular frequencies from Laplace-Lagrange linear theory, and noticed that the $\nu_{6}$ secular resonance (though he did not use this name) was adjacent to the Flora family. His work was the first, that we know of, to notice the possible interaction of secular resonances with asteroid families. Values of secular frequencies for asteroids and planets were also obtained by (Brouwer and van Woerkom, 1950) using a linear theory of secular perturbations, substantially improved by the introduction of the second order terms in perturbing mass accounting for the 5:2 and 2:1 near commensurabilities of Jupiter and Saturn. (Williams, 1969) used a non-linear semi-analytic

\footnotetext{
* Corresponding author.

E-mail addresses: valerio.carruba@unesp.br (V. Carruba), vokrouhl@cesnet.cz (D. Vokrouhlický), bojan@matf.bg.ac.rs (B. Novaković).
} 
theory that used the Gaussian method to treat short periodic perturbations to analyze the orbital evolution of asteroids over large periods of time. Two years later, the same author identified the linear secular resonances in the main belt as an effective mechanism for the depletion of asteroids. Our understanding of the dynamics in linear secular resonances greatly improved later on thanks to analytic models of the $\nu_{6}$ and other resonances, (Yoshikawa, 1987; Knežević et al., 1991; Morbidelli and Henrard, 1991; Morbidelli, 1993), when, using Hamiltonian formalisms, equilibrium points and the phase space of the resonances were first investigated.

Knowledge on the orbital position of the asteroid families themselves advanced significantly in the years 1990s, thanks to a seminal series of papers by Milani and Knežević, who studied how to analytically (Milani and Knežević, 1990) and numerically (Knežević and Milani, 2000, 2003) determine proper elements, conserved quantities of the motion over timescales of Myr. These authors also first investigated non-linear secular resonances (Milani and Knežević, 1992, 1994) and studied how they affected values of proper elements, when purely gravitational forces were considered. A modern theory of proper elements for high-inclination orbits in the main belt, and the Hungaria region, was initiated by works of Lemaitre and Morbidelli (Lemaitre and Morbidelli, 1994; Lemaitre et al., 1994).

It was, however, in the years 2000s, that the dynamical importance of the Yarkovsky-driven dynamical evolution of asteroids into secular resonance was first revealed. The Yarkovsky effect is a thermal radiation force that causes objects to undergo semi-major axis drift as a function of their size, spin, orbit, and material properties (Vokrouhlický et al., 2015). The seminal study of (Bottke et al., 2001) on the Yarkovsky driven mobility of asteroid showed that the shape of the Koronis family in the $(a, e)$ proper domain can only be explained if asteroids migrating toward higher semi-major axis interacted with the pericenter resonance $g+$ $2 g_{5}-3 g_{6}$ and, as a consequence, had their eccentricity increased because of the passage through the resonance. Several other papers investigated the role of the interplay of the Yarkovsky effect with secular resonances, among these one can quote the work on the Eos family and the $z_{1}$ secular resonance (Vokrouhlický and Brož (Vokrouhlický et al., 2002), Vokrouhlický et al. (2006a), interestingly enough (Brouwer, 1951) argued that the Eos family had to be young, because of the clustering of the secular angles of the then known family members (Vokrouhlický et al., 2006a). showed that this was a consequence of the Yarkovsky-induced evolution of asteroids into the $z_{1}$ resonance), on the Sylvia family and the $z_{1}$ resonance (Vokrouhlický et al., 2010), and of V-type objects and the $z_{2}$ secular resonance (Carruba et al., 2005). Carruba et al. (2014b) studied the interaction of V-type photometric candidates with the $g+$ $g_{5}-2 g_{6}$ secular resonance, a $g$-type resonance, in particular for the region of the Astraea family. Recently (Milani et al., 2017), obtained resonant proper element adapted to this resonance and an age estimate for this family, among others.

Of particular interest for the topic of this work was the identification of the resonant nature of the Agnia (Vokrouhlický et al., 2006b) and Tina (Carruba and Morbidelli, 2011) families. These were the first two families to have a majority or all members in librating states of the $z_{1}$ (Agnia) and $\nu_{6}$ secular resonances. Conserved quantities of secular dynamics can be used to set independent constraints on the magnitude of the original ejection velocity field of these peculiar families. For the case of the $\nu_{6}$ secular resonance, objects in anti-aligned librating states [37, or in paradoxal libration, according to 19], can be prevented to achieve high values of eccentricity, and remain long-term stable, as is the case for members of the Tina family.

Other developments in the 2000s involved the use of frequency domains such as the $(n, g, g+s)$ for family determination purposes (Carruba and Michtchenko, 2007, 2009), and for visualization of secular resonances in frequency domains, as also previously done by other authors (Milani and Knežević, 1994). The complicated three-dimensional structure of secular resonances in the $(a, e, \sin i)$ appears as lines in appropriate frequency domains, making it easier to visualize and inspect asteroid families affected by these resonances. The late 2000 s also showed that secular resonances with terrestrial planets are important in the Hungaria (Warner et al., 2009; Milani et al., 2010) and inner main belt region, for the Vesta (Carruba et al., 2005) and Phocaea (MilićŽitnik and Novaković, 2015) regions.

Among the most recent highlights was the discovery of the dynamical importance of secular resonances with massive bodies other than planets, mostly, but not only, with (1) Ceres (Novaković et al., 2015; Tsirvoulis and Novaković, 2016). The orbital distribution in the $(a, \sin i)$ domain of members of the Hoffmeister and Astrid families was for the first time explained by the effect of nodal resonances $s-s_{C}$ with Ceres. Finally, by modifying the value of inclination of family members, and, indirectly, of the $v_{W}$ component of the observed ejection velocity field, nodal secular resonances with massive asteroids or dwarf planets, such as the $s-s_{C}$ secular resonance with Ceres, can cause $v_{W}$ to become more and more leptokurtic, i.e., more peaked and with larger tails than that of a Gaussian distribution. By simulating fictitious asteroid families and by requiring that the current value of the Pearson kurtosis of $v_{W}, \gamma_{2}\left(v_{W}\right)$, be attained, independent constraints on the value of families ages can be obtained for families affected by these kinds of resonances (Carruba, 2016; Carruba et al., 2016b).

In this work we briefly review some of the most recent results on asteroid families affected by secular resonances, with a focus on asteroid families with a majority of their members inside a secular resonance and families affected by secular resonances with Ceres or massive asteroids.

\section{Secular dynamics}

Secular resonances are commensurabilities that involve the frequencies of the asteroid longitude of perihelion $g$, node $s$ and the fundamental frequencies of planetary theories $g_{i}=\langle\dot{\varpi}\rangle$ and $s_{i}=\langle\dot{\Omega}\rangle$, where $i$ is a suffix that indicates the planets ( 5 for Jupiter, 6, for Saturn, etc.), and the symbols \langle\rangle indicate long-term mean value of the corresponding frequency. Interested readers can look (Knežević and Milani, 2000, 2003) for more details on the Fourier transform methods normally used to numerically obtaining values of planetary and asteroid frequencies. In order to be in a secular resonance, the proper frequencies have to satisfy the relationship:

$p \cdot g+q \cdot s+\sum_{i}\left(p_{i} \cdot g_{i}+q_{i} \cdot s_{i}\right)=0$,

where the integers $p, q, p_{i}, q_{i}$ have to fulfill the D'Alembert rules of permissible arguments: the sum of the coefficients must be zero and the sum of coefficients of nodal longitudes frequencies must be even. The combinations (Eq. (1)) that only involve the frequency of the asteroid perihelion are often referred to as pericenter resonances, while those with only frequency of the asteroid node are named as node resonances.

The most important linear secular resonances in asteroid dynamics occur when $\nu_{6}=g-g_{6}=0 \operatorname{arcsec} / \mathrm{yr}, \nu_{5}=g-g_{5}=0 \operatorname{arcsec} / \mathrm{yr}$, and $\nu_{16}=s-s_{6}=0$ arcsec/yr. Pericenter resonances affect an asteroid eccentricity, while node resonances influence an asteroid inclination. Nonlinear secular resonances involve higher order commensurabilities, as for the $z_{k}=k\left(g-g_{6}\right)+s-s_{6}$ resonances sequence, that was first investigated by Milani and Knežević, 1992, 1994 and later confirmed by Vokrouhlický et al., 2006a, 2006b, Carruba (2009), Carruba et al. (2015), among others. While mean-motion resonances have a characteristic $\mathrm{V}$ shape in the $(a, e)$ plane, secular resonances usually have complicated three-dimensional structures in the proper $(a, e, \sin i)$ space and cannot easily be drawn in a plane. Fig. 1 shows the locations of the $\nu_{6}$ and $z_{k}$ resonances, of the pericenter resonance $g-2 g_{6}+g_{5}$, and of the node resonance $s-s_{6}-g_{5}+g_{6}$ determined for proper $e=0.10$ (the two curves refer to resonances computed for values of $e$ to within \pm 0.025 of the central value) with method of (Milani and Knežević, 1994) using the values of planetary frequencies listed in Table 1 of (Carruba and Michtchenko, 2007). The location and shape of the secular resonances change significantly for 


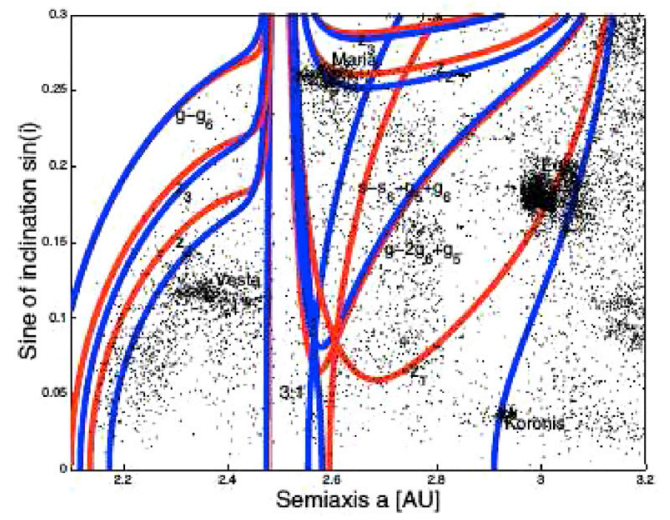

Fig. 1. Location of some of the main secular resonances in the asteroid belt. The secular resonances obtained for the fixed value 0.10 of the proper eccentricity are superimposed to the proper elements $a$ and $\sin i$ of the asteroids with proper e within the range of \pm 0.025 of the central value. The names indicate the main asteroid families in the region. The pairs of red and blue lines display the locations of the edges of the resonances. See Figs. 7-9 of (Milani and Knežević, 1994) for more details. (For interpretation of the references to colour in this figure legend, the reader is referred to the Web version of this article.)

\section{Table 1}

Age estimates and values of the $V_{E J}$ parameter for the families for which the $v_{W}$ approach has been used so far. The first column displays the Family Identification Number (FIN), as from (Nesvorný et al., 2015), the second column the family name, the third the current value of $\gamma_{2}\left(v_{W}\right)$, as from (Carruba and Nesvorný, 2016), the fourth the estimated family age, and the fifth the estimated value of the $V_{E J}$ parameter.

\begin{tabular}{|c|c|c|c|c|}
\hline \multirow[t]{2}{*}{ FIN } & Family & Current & Estimated & Estimated \\
\hline & Name & $\gamma_{2}\left(v_{W}\right)$ & age [Myr] & $V_{\mathrm{EJ}}[\mathrm{m} / \mathrm{s}]$ \\
\hline 515 & 1128 Astrid & 3.69 & $140 \pm 30$ & $10_{-10}^{+30}$ \\
\hline 518 & 1644 Rafita & 0.63 & $480 \pm 15$ & $20_{-15}^{+30}$ \\
\hline 802 & 148 Gallia & 2.02 & $630_{-70}^{+30}$ & $5_{-5}^{+17}$ \\
\hline 803 & 480 Hansa & 0.81 & $460_{-360}^{+280}$ & $80_{-65}^{+10}$ \\
\hline 805 & 945 Barcelona & 1.48 & $265_{-35}^{+45}$ & $15_{-15}^{+20}$ \\
\hline
\end{tabular}

different values of the proper $e$, making it difficult to visualize how asteroid families members may interact with such resonances.

The reason for this is that in the $(a, e, \sin i)$ space the position of a resonance depends on all three elements. To overcome this problem, one can project asteroids in different domains, like that of the proper frequencies $(n, g, s)$, where the mean-motion and secular resonances are separable. ${ }^{1}$ In this space, the location of mean-motion resonances mostly depends on the mean-motion proper frequency $n$ (see (Knežević and Milani, 2000) for a discussion on how to compute this quantity), while the position of secular resonances only depends on the proper $(g, s)$ frequencies and appear as lines in a plane.

Different planes can be used to better represent asteroids interacting with different type of secular resonances. $g$-type secular resonances could be better displayed in an $(n, g)$ or $(a, g)$ plane, $s$-type resonances would be better displayed in $(n, s)$ or $(a, s)$ planes, while $(g+s)$-type of resonances could be better shown in a plane in which one of the axis displays $g+s$. Fig. 2 displays a projection of main belt asteroids in the $(g, g+s)$ domain from (Carruba and Michtchenko, 2007). Other type of representations for different type of non-linear secular resonances, such as $2 g+s, 3 g+s, g-$ $s$ and other, were introduced and explored in (Carruba and Michtchenko, 2009).

\footnotetext{
1 According to nonlinear perturbation theories, the proper frequencies are functions of the proper elements and the transformation from proper elements to proper frequencies is one-to-one if the Kolgomorov's non-degeneracy condition is satisfied (Ferraz-Mello, 2007).
}

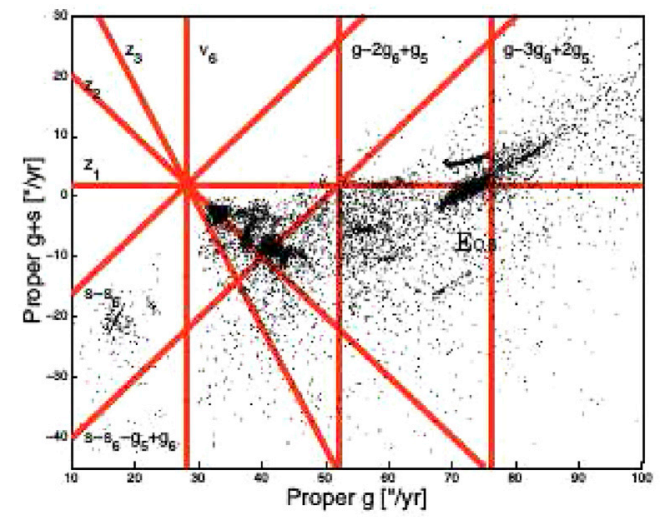

Fig. 2. A projection of asteroids in the $(g, g+s)$ domain. The horizontal line displays the location of the $z_{1}=g-g_{6}+s-s_{6}$ secular resonance. From Fig. 6 of (Carruba and Michtchenko, 2007).

Asteroids interacting with secular resonances can also be better identified in frequency domains. The libration region of each secular resonance has a typical width around the respective combination of planetary frequencies. For instance, it was shown that asteroids in librating states of the $\nu_{6}$ secular resonance could be found to within \pm 1.2 arcsec/year from $g=g_{6}$ for asteroids in the region of the Tina and Euphrosyne families (Carruba and Morbidelli, 2011; Machuca and Carruba, 2012), while the limit for asteroids in librating states of the $z_{1}$ resonance was \pm 0.3 arcsec/year for $g+s=g_{6}+s_{6}$ for asteroids in the Padua family region (Carruba, 2009). By selecting a cutoff value in frequencies it is therefore possible to select objects that are likely to be in librating states of a given resonance. Such objects, or likely resonators, could then be investigated to check if their resonant argument is actually librating around an equilibrium point, so as to determine the effective size of the population of objects in actual resonant configuration. The likely resonator criteria is, therefore, an useful tool in pre-selecting objects more likely to be affected by secular dynamics.

Asteroid families interacting with secular resonances can also be identified in domains of proper frequencies rather than proper elements. In the Hierarchical Clustering Method of (Zappalà et al., 1990), asteroid families are identified with the following procedure: given an individual asteroid the distance between this object and the other one is computed. If the distance is less than a threshold limit $\left(d_{\text {cutoff }}\right)$, the new object is added to the list. The procedure is repeated until no new family member is found. A critical point in this procedure is related to the choice of a reasonable metric in the three-dimensional element space. In (Zappalà et al., 1990), the distance is defined as

$d_{1}=n a \sqrt{k_{1}(\Delta a / a)^{2}+k_{2}(\Delta e)^{2}+k_{3}(\Delta \sin i)^{2}}$,

where $n$ is the asteroid mean motion; $\Delta x$ the difference in proper $a, e$, and sini; and $k_{1}, k_{2}, k_{3}$ are weighting factors, defined as $k_{1}=5 / 4, k_{2}=2, k_{3}=$ 2 . Other choices of weighting factors are possible and yield similar results. (Carruba and Michtchenko, 2007) looked for families that interacted with $g+s$-type secular resonances in a $(n, g, g+s)$, such as the Eos domain using a distance metric of the form:

$d_{2}=\sqrt{h_{1}\left(\Delta n / h_{0}\right)^{2}+h_{2}(\Delta g)^{2}+h_{3}(\Delta(g+s))^{2}}$

where $h_{0}$ is a normalization factor of dimension $1^{\circ} / \operatorname{arcsec}$, and the simplest choice for the $h_{i}(\mathrm{i}=1,3)$ weights is to take them all equal to 1 . The distance in frequency space then has the units of arcsec/yr. The method looking for families in frequency domains was called Frequency Hierarchical Clustering Method, of FHCM, by these authors. Families found in this domain were, for appropriate choices of $d_{2}$, able to connect to objects that drifted in secular resonances of $g+s$-type and that were 
not recognized as family members by the traditional HCM in proper element domain.

Fig. 3 displays a $(g, g+s)($ panel A) and a $(a, \sin i)($ panel B) projection of the Eos family obtained with the classical HCM (green dots), and with the frequency HCM (blue crosses), as from Carruba and Michtchenko (2007). Secular resonances up to order 6, i.e., those resonances for which the sum of the coefficients in the resonant argument does not exceed 6, are shown in the $(a, \sin i)$ plane and are identified by thicker lines in the $(g, g+s)$ plane. Among other results, the Eos family identified in the HCM domain was able to connect to four asteroids (20845) 2000 UY102, (21211) 1994 PP36, (33780) 1999 RU171, and (62948) 2000 VE32, currently inside the $z_{1}$ resonance and at low values of proper $e$ and $\sin i$ (Vokrouhlický et al., 2006a). hypothesized that those objects were former members of the Eos family that diffused to their current position due to the interplay of the Yarkovsky effect and the $z_{1}$ resonance, and this was later confirmed by subsequent taxonomic analysis of these asteroids that showed that these objects are of the same, peculiar type of most of the Eos family asteroids, the $\mathrm{K}$ type, and therefore most likely to have originated from this group. Other objects diffusing in $g, s$ and other types of secular resonances were also identified by the FHCM, but not by the standard HCM.

Other families interacting with other types of secular resonances, such as $s, g, g+s, g-s, 2 g+s$, and $3 g+s$, and the appropriate distance metrics to study each case were also investigated in (Carruba and Michtchenko, 2007, 2009). Interested readers can found additional information in those papers.

\section{Families interacting with first-order secular resonances}

Once asteroids interacting with secular resonances have been identified, using methods described in the previous section, information from secular dynamics can then be used to set constraints on the dynamical evolution of asteroid families interacting with secular resonances. Here we will review some of the results of the last decade on families in linear and non-linear secular resonances.

Among linear resonances, the $\nu_{6}=g-g_{6}$ resonance is one of the main effective mechanisms for increasing an asteroid eccentricity, and one of the main source of NEA (Morbidelli and Henrard, 1991; Bottke et al., 2002; Granvik et al., 2016). This resonance interacts with the Tina (Carruba and Morbidelli, 2011) and Euphrosyne (Carruba et al., 2014a) families, and sets the boundary for highly inclined objects in the central and outer main belt (Carruba, 2010). Fig. 4 displays an $(a, \sin i)$ projection of the location of the Tina and Euphrosyne families.

The resonance topology depends on the distance of the asteroid with respect to Saturn. Fig. 5 displays the phase space of equi-Hamiltonian curves for first-order resonances, as shown in the classical work of (Ferraz-Mello, 1985). For first order resonances, at higher distances from

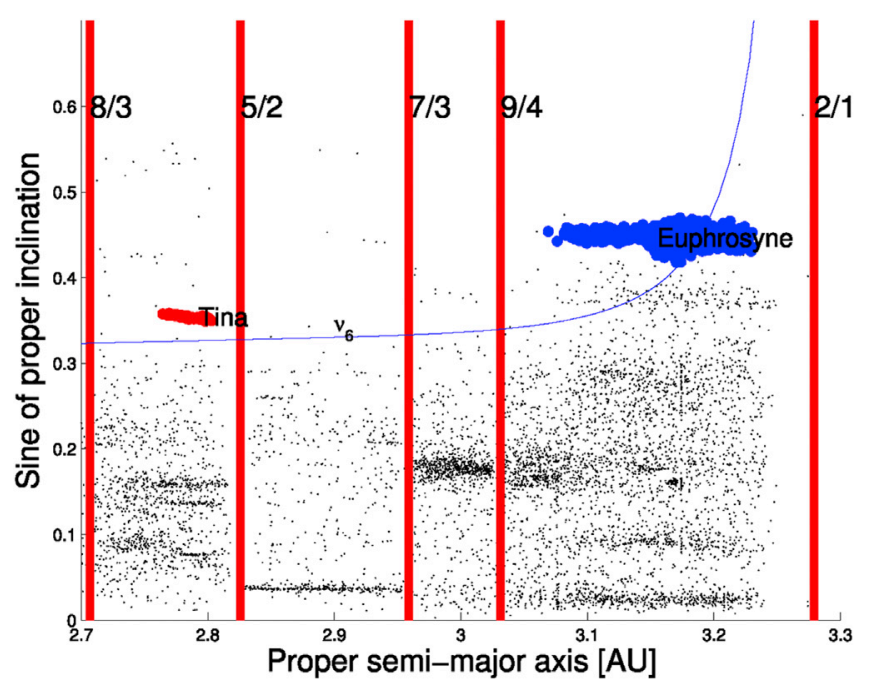

Fig. 4. The orbital location in the $(a, \sin i)$ plane of the Tina (red dots) and Euphrosyne (blue dots) families. The location of the $\nu_{6}$ resonance was computed for the eccentricity value of (1222) Tina by using the second-order and fourthdegree secular perturbation theory of (Milani and Knežević, 1992). Vertical red lines displays the location of the main mean-motion resonances in the region. (For interpretation of the references to colour in this figure legend, the reader is referred to the Web version of this article.)
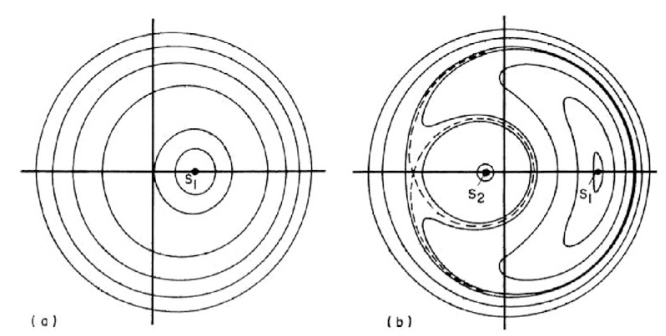

Fig. 5. The phase space in the $(e \cos \sigma, e \sin \sigma)$ domain of a first-order resonance far (panel a) and near (panel b) the perturbing objects. Figure adapted from (Ferraz-Mello, 1985).

the perturber, which for the $\nu_{6}$ resonance would correspond to Saturn and the inner main belt, the phase space of the resonance would have an equilibrium point at $0^{\circ}$, and two possible types of orbits: libration around the equilibrium point, for which the resonant argument of the resonance will oscillates near $0^{\circ}$, and circulation, for which the resonant argument will cover all possible range of values. The curve separating these two classes of orbits is called the separatrix. This type of dynamical behavior
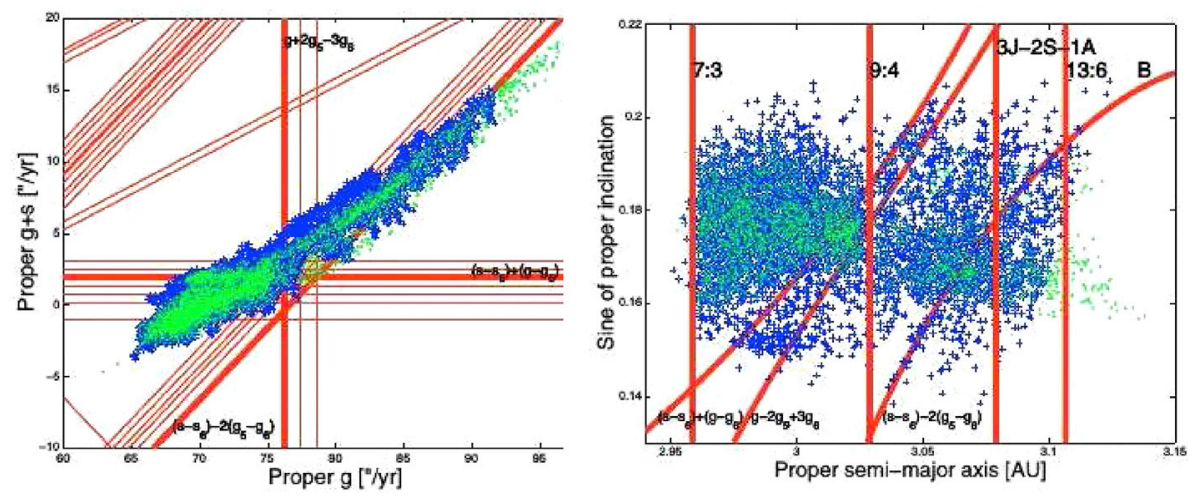

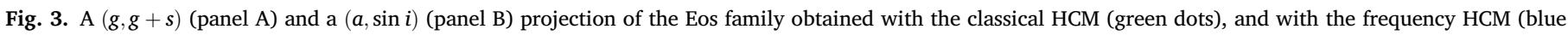

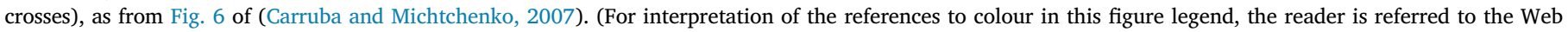
version of this article.) 

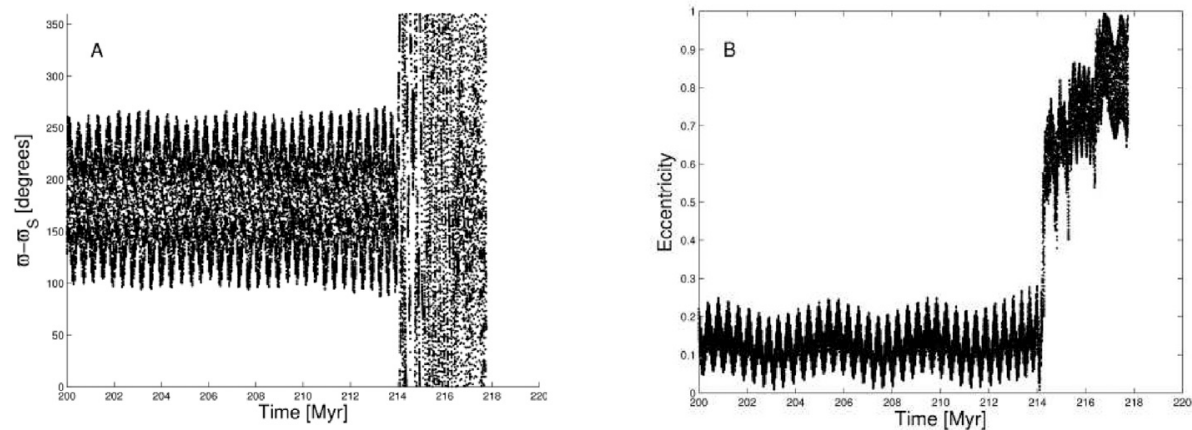

Fig. 6. Panel A: the time-evolution of the osculating resonance argument for a clone of the asteroid (211412) (2002 VL103). Panel B: the time-evolution of the osculating eccentricity of the same test particle. From Fig. 7 of (Carruba and Morbidelli, 2011).

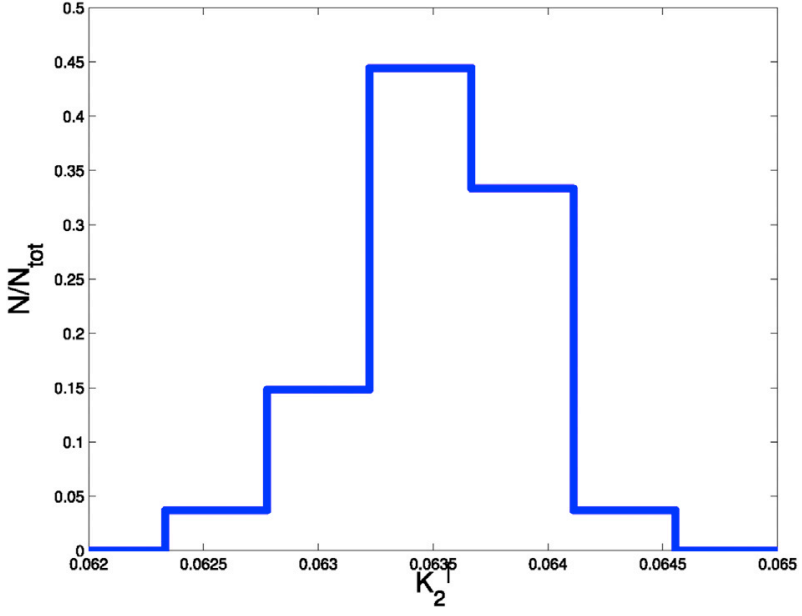

Fig. 7. The current distribution of the $K_{2}^{\prime}$ quantity for Tina family members. From Fig. 10 of (Carruba and Morbidelli, 2011).

$V_{\mathrm{SD}}=V_{\mathrm{EJ}}\left(\frac{5 \mathrm{~km}}{D}\right)$,

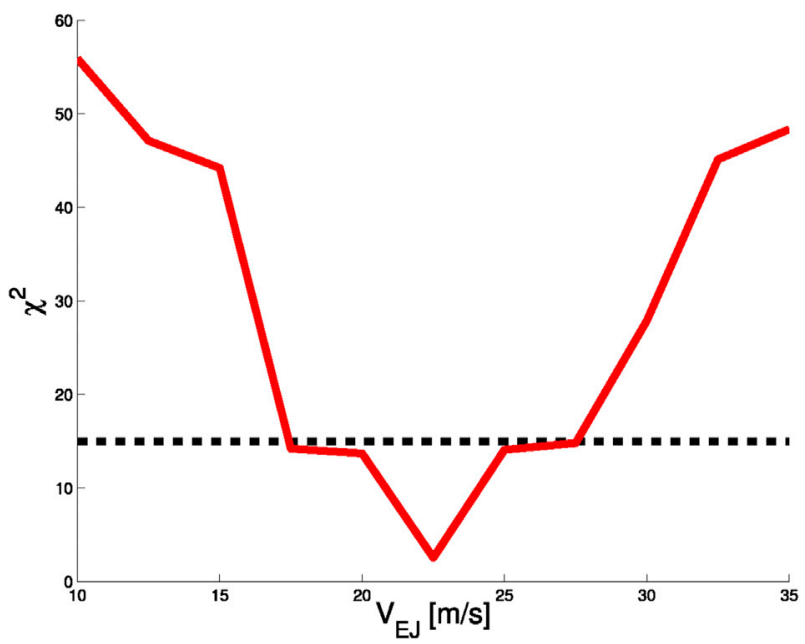

Fig. 8. The values of $\chi_{2}$ of the synthetic families as a function of the values of $V_{\mathrm{EJ}}$. The horizontal line displays the probability confidence level. From Fig. 11 of (Carruba and Morbidelli, 2011).

is shown in Fig. 5, panel A. Closer to the perturbing planet, however, another point of equilibrium at $180^{\circ}$ may appear, and the separatrix may

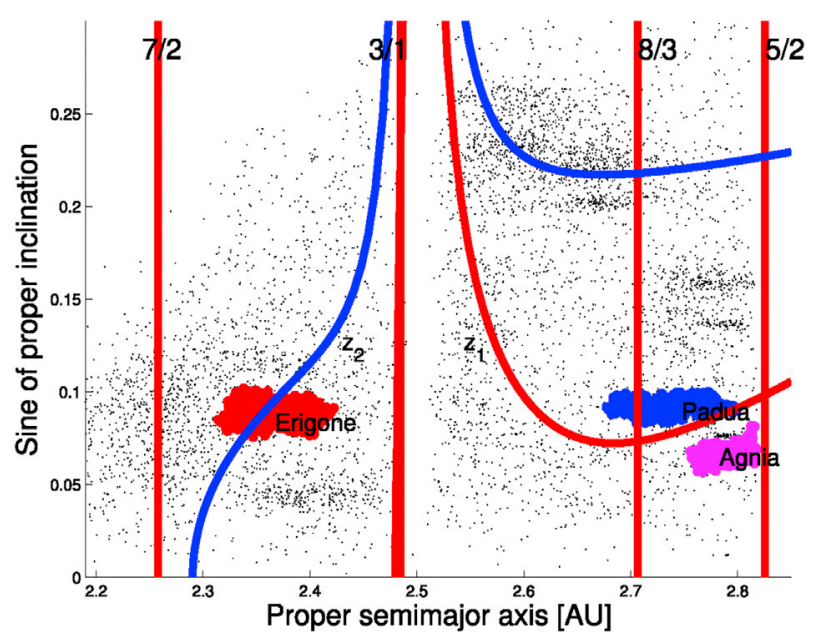

Fig. 9. The orbital location of the Padua, Agnia, and Erigone family and their position with respect to the $z_{1}$ and $z_{2}$ secular resonances.

form a loop around this equilibrium point. Apart from orbit of libration near $0^{\circ}$ and circulation, a new class of orbits may be possible in these regions: the resonant argument may librate around $180^{\circ}$ (Ferraz-Mello, 1985). calls this type of orbits paradoxical librators, while other authors (Morbidelli and Henrard, 1991) define them as anti-aligned librators. The latter name is justified by the fact that, for the $\nu_{6}$ resonance, the argument of pericenter of Saturn and of the perturbed object are roughly separated by $180^{\circ}$. These orbits are not really on libration, but rather are circulating orbits trapped by the separatrix loop.

The Tina family is the only asteroid family currently known whose members are all in anti-aligned states of the $\nu_{6}$ secular resonance (Carruba and Morbidelli, 2011). This orbital configuration protects the family members from reaching planetary-crossing orbits. Since anti-aligned orbits cannot cross the separatrix, the maximum eccentricity that an orbit can reach is limited, and may not reach planet-crossing levels. This behavior is shown in Fig. 6 for a clone of the asteroid (211412) (2002 VL103), a member of the Tina family. As long as the asteroid remains inside the anti-aligned librating configuration, oscillations in eccentricity are limited. Once the asteroid escapes the stable region and goes into a circulating orbit, changes in eccentricity grows dramatically and $e$ reaches Mars-crossing levels of 0.4 in short time-scales. All Tina family members are therefore found in a stable island of the $\nu_{6}$ secular resonance.

The resonant nature of the Tina family allows to obtain information on the original ejection velocity field of this group, not available for families not affected by secular resonances. At the simplest level of perturbation theory, the $\nu_{6}$ resonance is characterized by the conserva- 
tion of the quantities $K_{1}=\sqrt{a}$ and $K_{2}=\sqrt{a\left(1-e^{2}\right)}(1-\operatorname{cosi})$. The quantity:

$K_{2}^{\prime}=\frac{K_{2}}{K_{1}}=\sqrt{1-e^{2}}(1-\cos i)$,

is preserved even when the Yarkovsky force is accounted for (Carruba and Morbidelli, 2011). The current distribution of the $K_{2}^{\prime}$ quantity, shown in Fig. 7 for Tina asteroids, therefore preserves information on the original one. If we assume that the ejection velocity field that created a Tina family was isotropic, Gaussian, centered around 0 and with a size-dependent standard deviation that follows the relationship:where $D$ is the asteroid diameter in $\mathrm{km}$, and $V_{\mathrm{EJ}}$ is a free parameter, usually of the order of the estimated escape velocity from the parent body, we can then best-fit the currently observed $K^{\prime}{ }_{2}$ values with those of simulated asteroid families with different $V_{\mathrm{EJ}}$ parameters. For each family, we introduce a $\chi^{2}$-like variable defined as:

$\chi^{2}=\sum_{i=1}^{N_{\text {int }}} \frac{\left(q_{i}-p_{i}\right)^{2}}{q_{i}}$,

where $N_{\text {int }}$ is the number of interval used for the values of $K_{2}^{\prime}, q_{i}$ is the number of real objects in the $\mathrm{i}$-th interval in $K^{\prime}{ }_{2}$ and $p_{i}$ is the number of synthetic family members in the same i-th interval. Fig. 8 shows how the value of $\chi_{2}$ changes with the value of $V_{\mathrm{EJ}}$ adopted to generate the synthetic family. The minimal value of $\chi_{2}$ (best fit) is obtained for $V_{\mathrm{EJ}}=22 \pm$ $5 \mathrm{~m} / \mathrm{s}$, in good agreement with the $20.0 \pm 2.5 \mathrm{~m} / \mathrm{s}$ estimate obtained from Yarko-YORP methods (Vokrouhlický et al., 2006c). This method can also be applied to families in non-linear secular resonances, which will be the subject of the next section of this paper.

\section{Families in non-linear secular resonances}

There are asteroid families interacting with non-linear secular resonances of the $z_{k}$ sequence. More than $75 \%$ of the members of the Agnia (Vokrouhlický et al., 2006b) and Padua (Carruba, 2009) families are on librating states of the $z_{1}=g-g_{6}+s-s_{6}$ resonance, while $14 \%$ of the members of the Erigone family are in librating states of the $z_{2}=$ $2\left(g-g_{6}\right)+s-s_{6}$ resonance (Carruba et al., 2015). The Agnia family, which is now know to have the sub-family of (3395) Jitka (Spoto et al., 2015), was the first group identified to have the majority of its members in librating states of a non-linear secular resonance. The orbital position of these families and their relative location with respect to the appropriate $z_{k}$ resonances is shown in Fig. 9.

It is possible to show that the $K_{2}^{\prime}\left(z_{1}\right)$ quantity below is preserved for the $z_{1}$ secular resonance (Vokrouhlický et al., 2006b; Carruba, 2009):

$K_{2}^{\prime}=\frac{K_{2}}{K_{1}}=\sqrt{1-e^{2}}(2-\cos i)$,

By performing an analysis similar to that discussed in Sect. 3 for the Tina family it can be shown that the value of the ejection velocity parameter compatible with the $K_{2}^{\prime}\left(z_{1}\right)$ distribution is $15 \pm 5 \mathrm{~m} / \mathrm{s}$ for the Agnia family (Vokrouhlický et al., 2006b) and $35 \pm 8 \mathrm{~m} / \mathrm{s}$ for the Padua one (Carruba, 2009).

Concerning other families (Carruba et al., 2015), showed that the $K^{\prime}{ }_{2}\left(z_{2}\right)=K^{\prime}{ }_{2}=K_{2} / K_{1}=\sqrt{1-e^{2}}(3-\cos i)$ quantity is not preserved for asteroids members of the Erigone family. Yet, it is possible to obtain information on the age of this group from secular dynamics. Assuming that the current population of $z_{2}$ librators is in steady-state, one can use this information to set lower limits on the Erigone family age.

Carruba et al. (2015) generated a fictitious Erigone family with optimal $V_{\mathrm{EJ}}$ parameter, as found with Yarko-YORP methods, and integrated this group over $400 \mathrm{My}$, beyond the maximum possible value of the age of this group. They then analyzed the resonant argument $\sigma$ of the $z_{2}$ secular resonance for all simulated particles. The behavior of this angle could be quite complex: because of the drift in semi-major axis caused by non-gravitational forces, asteroids can be alternate between times in which they are captured into resonance, and times in which they escape to circulating orbits, and vice-versa. Fig. 10 displays the time dependence of the $z_{2}$ resonant argument of a simulated particle that alternated between phases of libration and circulation in the $z_{2}$ secular resonance. Overall, while the number of $z_{2}$ librators changes with time, if the population of librators from the Erigone family is in a steady-state, this number should fluctuate around the median value, with fluctuation of the order of one standard deviation. A similar behavior was observed for the population of asteroids currently inside the M2:1A mean-motion resonance (Gallardo et al., 2011). The minimum time needed to reach a steady-state could therefore be used to set lower limits on the age of the Erigone family.

Carruba et al. (2015) analyzed the resonant angle of all simulated particles, and computed the fraction of family members in $z_{2}$ resonant states as a function of time. Fig. 11 displays the results: the number of $z_{2}$ librators fluctuates with time, but reaches its median value after $125 \mathrm{My}$ (after $\simeq 90$ My if we consider the median value plus or minus the standard deviations as an estimate of the error, blue dashed lines in Fig. 11). This sets a lower limit on the family age, compatible with estimates from the literature that yield an age older than $150 \mathrm{Myr}$ for this group.

Recently, a new class of secular resonances, involving Ceres and massive asteroids as pertubers, has been identified (Novaković et al., 2015). Their effect on the dynamical evolution of asteroid families will be discussed in the next section.

\section{Secular resonances with Ceres and massive asteroids}

The importance of secular resonances with massive asteroids has been recently realized by (Novaković et al., 2015). In particular, these authors found that the spreading in orbital inclination seen in the Hoffmeister family, is a consequence of the nodal liner secular resonance with Ceres, namely $\nu_{1 C}=s-s_{C}$. It was actually shown that passing through the $\nu_{1 C}$ resonance may cause significant changes in the orbital inclination of an asteroid. This effect is visible in Fig. 12, where the time evolution of the inclination and of the resonant angle of the $\nu_{1 C}$ resonance, for an asteroid belonging to the Hoffmeister family, are plotted. The instant of increase in inclination corresponds very well to the libration period of the resonant angle.

This was the first direct proof that a secular resonance between Ceres and other asteroids, prior completely overlooked, can cause significant orbital evolution of the latter. The result opened several new possibilities to investigate the dynamics of small bodies, and initiates a series of related studies.

One of the most interesting works in this respect was performed by (Carruba et al., 2016a). The authors showed that secular resonances with Ceres may explain why there is no Ceres family, showing that methods for identifying asteroid family based on a search of neighbors pairs, like the HCM, cannot reveal the possible existence of a Ceres family. In order to highlight a role of Ceres (Carruba et al., 2016a), obtained synthetic proper elements dynamical map for the region of the central main belt without and with Ceres as a perturber. Results are shown in Fig. 13.

As observed in Fig. 13, panel B, accounting for Ceres causes the appearance of a 1:1 mean-motion resonance with this dwarf planet. Most importantly, linear secular resonances of nodes $s-s_{C}$ and pericenter $g-$ $g_{C}$, first detected by (Novaković et al., 2015), significantly destabilize the orbits in the proximity of Ceres. Combined with the long-term effect of close encounters with Ceres, this has interesting consequences for the survival of members of the Ceres family in the central main belt. Not many family members are expected to survive near Ceres, and this would cause significant difficulties in using standard dynamical family identification techniques, since they are based on looking for pairs of neighbors in proper element domains. Since the close neighbors of Ceres would have been removed on a short time-scale, only objects whose distance from Ceres is higher than the average distance between pairs of asteroids in the central main belt would have survived. Results of simulations of 


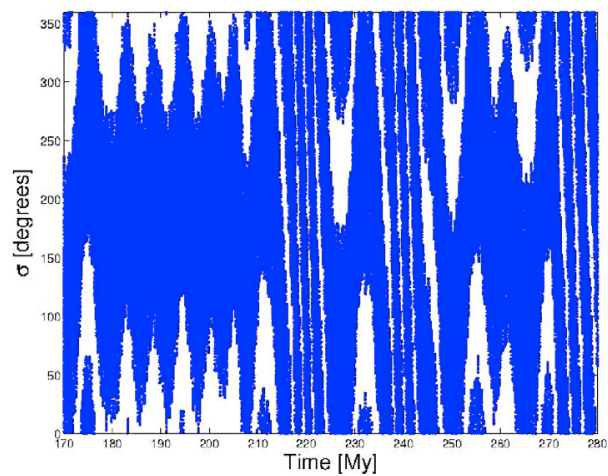

Fig. 10. Time behavior of the $z_{2}$ resonant argument of a simulated particle between 170 and 280 My. From Fig. 11 of (Carruba et al., 2015).

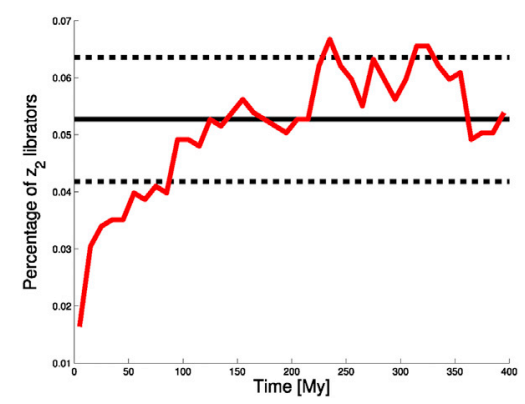

Fig. 11. Fraction of simulated Erigone family members in $z_{2}$ librating states as a function of time, normalized with respect to the median value. The horizontal blue line displays the median percentage of objects in the $z_{2}$ states, dashed lines display levels of median fraction plus or minus its standard deviation. From Fig. 12 of (Carruba et al., 2015). (For interpretation of the references to colour in this figure legend, the reader is referred to the Web version of this article.)
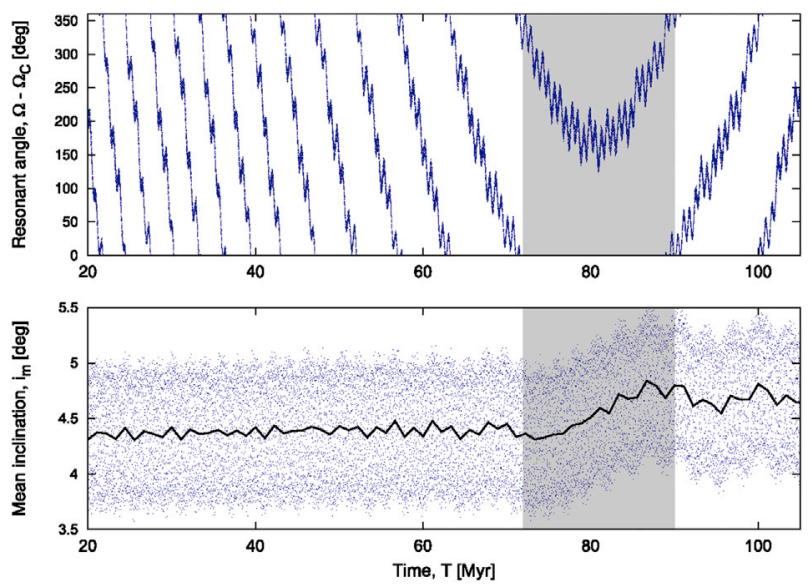

Fig. 12. Time behavior of the $\nu_{1 C}$ resonant argument (top panel) and of the mean orbital inclination (bottom panel), of a simulated particle located in the region of the Hoffmeister family. The time period in which the mean inclination is increasing for about $0.5^{\circ}$ is clearly related to the interval when the resonant argument is librating (shaded area). The solid black line denotes the average of the mean inclination to better appreciate the evolution.

fictitious Ceres families in (Carruba et al., 2016a) showed that secular dynamics would indeed clear the region near Ceres of neighbors, making it impossible to identify a Ceres family after timescales of $350 \mathrm{Myr}$.

Motivated by the aforementioned results (Tsirvoulis and Novaković, 2016), made a map of the locations of the linear secular resonances with
Ceres and Vesta in the asteroid belt, and investigated the magnitude of the perturbations. Their results show that in some cases the strength of secular resonances with Ceres and Vesta is similar to that of non-linear secular resonances with the major planets (Tsirvoulis and Novaković, 2016). have also identified several asteroid families crossed by the secular resonances with Ceres and Vesta, and pointed out that the post-impact evolution of these families may be significantly affected by aforementioned resonances. The list of these families includes the Astrid, Hoffmeister, Seinajoki and some other groups [see also (Novaković et al., 2016).

Secular dynamics with massive bodies can provide constraints on the ages of the " $v_{W}$ leptokurtic families", or families characterized by their interaction with secular resonances with Ceres, which will be the subject of the next section.

\section{6. $v_{W}$ leptokurtic asteroid families}

The change in inclination of a family members is related to the perpendicular component of the velocity at infinity $v_{W}$ through the corresponding Gauss equation:

$\delta i=\frac{\sqrt{1-e^{2}}}{n a} \frac{\cos (\omega+f)}{1+e \cos f} \delta v_{W}$

where the changes in proper $\delta i$ are computed with respect to the family center of mass, and $f$ and $\omega$ are the (generally unknown) true anomaly and perihelion argument of the disrupted body at the time of impact. If a family interacts with a secular resonance of node with a massive body, its inclination distribution will become more peaked and with larger tails, when compared with a Gaussian one. As a consequence, the value of the Pearson kurtosis of the $v_{W}$ component of the ejection velocity field, $\gamma_{2}\left(v_{W}\right)$, will also increase. (Carruba and Nesvorný, 2016) listed all families characterized by large values of this parameter. The minimum time needed to attain the current value of $\gamma_{2}\left(v_{W}\right)$ for the $v_{W}$ leptokurtic families can be used to set constraints on their ages. This method for dating $v_{W}$ leptokurtic families has been used, so far, to date six asteroid families, those of Astrid, Hoffmeister, Gallia, Barcelona, Hansa, and Rafita.

The Astrid family was the first family for which this method was applied (Carruba, 2016). This family, characterized by a squid-like appearance in the $(a, \sin i)$ domain (see Fig. 14, panel A), interacts with the $s-s_{C}$ resonance, which is responsible for its distribution in inclination.

By simulating various fictitious families and by demanding that the current value of the $\gamma_{2}\left(v_{W}\right)$ of the distribution in be reached over the estimated lifetime of the family of $140 \pm 30 \mathrm{Myr}$ (Carruba, 2016), showed that the thermal conductivity of Astrid family members should be $0.001 \mathrm{~W} \mathrm{~m}^{-1} \mathrm{~K}^{-1}$, unusual for a C-type family, and that the surface and bulk density should be higher than $1000 \mathrm{~kg} \mathrm{~m}^{-3}$. The time evolution of the $\gamma_{2}\left(v_{W}\right)$ for a family with the optimal parameters of the Yarkovsky force can be seen in Fig. 14, panel B.

Three of the most $v_{W}$ leptokurtic families are found in the highly inclined region of the central main belt: the Hansa, the Gallia, and the Barcelona families (Carruba et al., 2016b). used the $\gamma_{2}\left(v_{W}\right)$ approach to obtain family ages for these three families, whose results are summarized in Table 1. For the Gallia family, the current value of $\gamma_{2}\left(v_{W}\right)$ can only be reached over the estimated family age if Ceres is considered as a massive perturber. Fig. 15 shows the time behavior of this parameter for a scenario without (panel A) and with (panel B) Ceres as a massive body. Indeed, current values of $\gamma_{2}\left(v_{W}\right)$ can be reached over the estimated family age only if Ceres is considered as a massive body.

Finally, although this was not the main focus of their work (Aljbaae et al., 2016), applied the $v_{W}$ approach of family dating to the Rafita asteroid family, increasing the total number of families with age estimated in this way to five. All the results obtained so far with this family dating method are summarized in Table 1. 

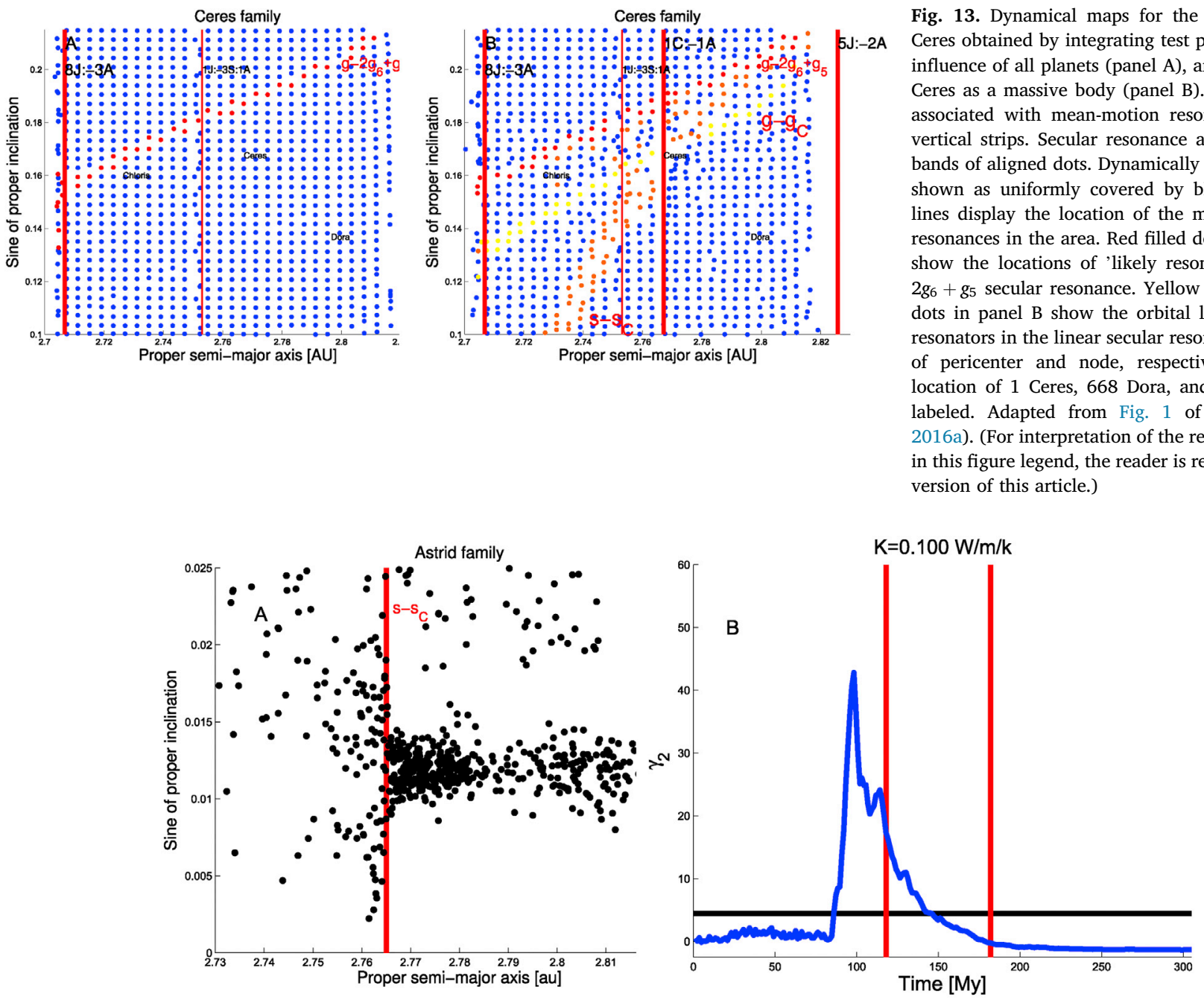

Fig. 14. Panel A: an $(a, \sin i)$ projection of members of the Astrid family. Vertical red lines display the location of local secular and mean-motion resonances. Panel B: the time evolution of the $\gamma_{2}\left(v_{W}\right)$ for a family with the optimal parameters of the Yarkovsky force. Vertical red lines displays the estimated age of the Astrid family, while the horizontal black line shows the current value of $\gamma_{2}\left(v_{W}\right)$ for this family. Adapted from figures of (Carruba, 2016). (For interpretation of the references to colour in this figure legend, the reader is referred to the Web version of this article.)
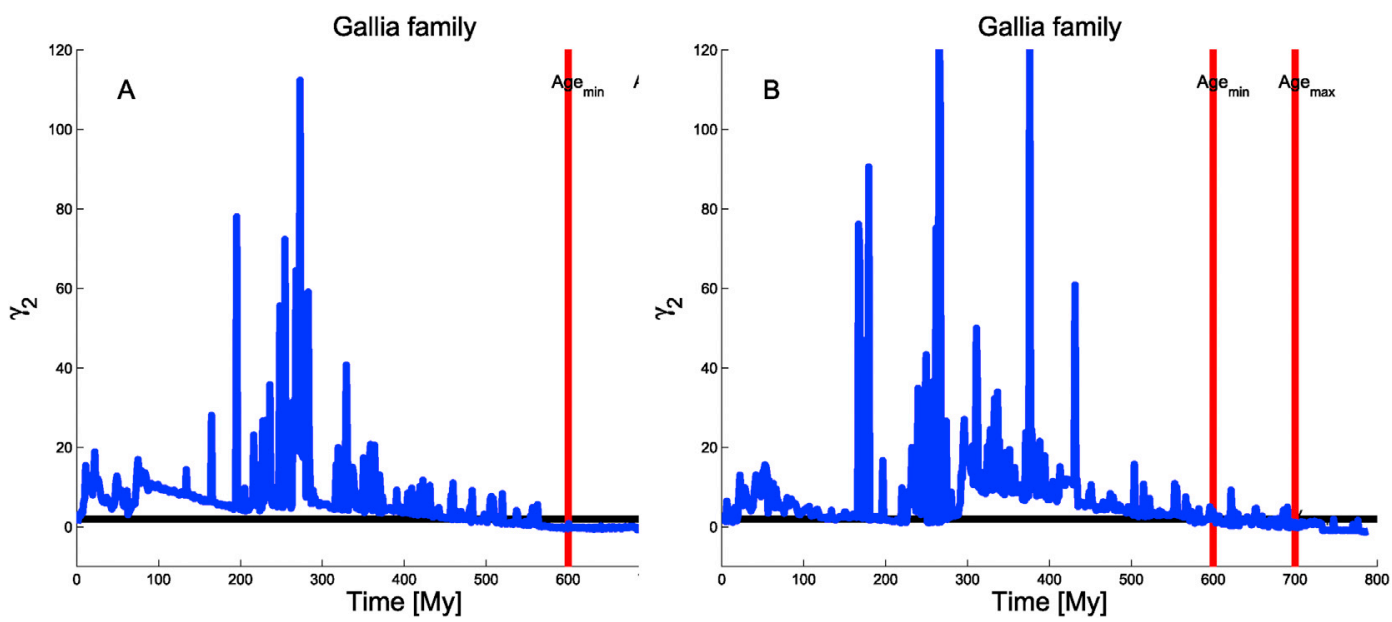

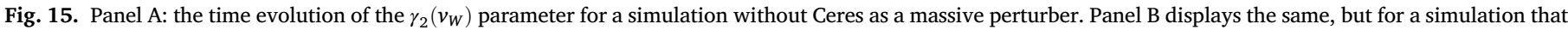

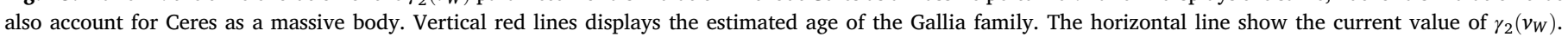

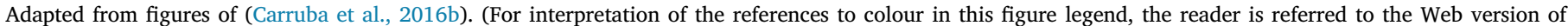
this article.) 


\section{Conclusions}

In this review paper, we saw how:

- Secular resonances have a complex structure in the proper $(a, e, \sin i)$ domain. But they appear as lines in proper $(n, g, s)$ domains. In particular $g$-type resonances should be plotted in a domain in which the y-axis is the $g$ frequency, $s$-type resonances will appear as horizontal lines in a plane in which the $s$ frequency is on the y-axis, and non-linear secular resonances should be plotted in a domain appropriate to their type $(g+s$ for $(g+s)$-type resonances, $g-s$ for $(g-s)$ resonances, etc.). By selecting a cutoff value near the combination of planetary frequencies for each resonance, it is possible to select asteroids more likely to be affected by secular dynamics, the so-called "likely resonators". Asteroid families interacting with secular resonances can also be identified in domains of proper frequencies (Carruba and Michtchenko, 2007, 2009).

- Because of their tilted shape in the space of proper orbital elements, secular resonances may effectively change proper eccentricity and/or inclination if the proper semi-major axis is evolving due to the Yarkovsky effect. This unique property has led to setting evidence of Yarkovsky effect influence in a number of asteroid families.

- Because of the preservation of quantities associated with the local secular dynamics, asteroid families interacting with linear (Tina family and the $\nu_{6}$ secular resonance) and non-linear (Agnia and Padua family and the $z_{1}$ resonance, the Erigone and the $z_{2}$ resonance) secular resonances still preserve information on their original ejection velocity field. The study of this families provides clues on the mechanisms of family formation not available for other, non-resonant families.

- The interaction of asteroid families with nodal secular resonances with Ceres may significantly change their inclination distribution, making it more leptokurtic, i.e., more peaked and with larger tails, when compared with a Gaussian one. By modeling the time evolution of the $v_{W}$ component of the ejection velocity field, it is possible to set constraint on the family age and original ejection velocity field of families for which the current value of the Pearson Kurtosis of $v_{W}$ is significantly larger than 0 . So far, six asteroids families, those of Astrid, Hoffmeister, Gallia, Barcelona, Hansa, and Rafita, have been dated with this method.

Overall, secular dynamics can provide invaluable hints for our understanding of the dynamical evolution of asteroid families, in many cases not available for non-resonant groups. This could be of potential great interest for the many new smaller families recently identified in (Nesvorný et al., 2015) and (Milani et al., 2014), whose resonant nature has yet to be investigated.

\section{Acknowledgments}

We are grateful to the two reviewers of this paper, an anonymous reviewer and Dr. Federica Spoto, for comments and suggestions that significantly improved the quality of this work. We would like to thank the São Paulo State Science Foundation (FAPESP) that supported this work via the grant 16/04476-8, and the Brazilian National Research Council (CNPq, grant 312313/2014-4). DV's work was funded by the Czech Science Foundation through the grant GA13-01308S. BN acknowledges support by the Ministry of Education, Science and Technological Development of the Republic of Serbia, project 176011. We acknowledge the use of data from the Asteroid Dynamics Site (AstDys) (http://hamilton.dm.unipi.it/astdys, (Knežević and Milani, 2003)).

\section{Appendix A. Supplementary data}

Supplementary data related to this article can be found at https://doi. org/10.1016/j.pss.2018.03.009.

\section{References}

Aljbaae, S., Carruba, V., Masiero, J., Domingos, R.C., Huaman, M., 2016. The Rafita asteroid family. MNRAS 467, 1016-1023.

Bottke, W.F., Vokrouhlický, D., Brož, M., Nesvorný, D., Morbidelli, A., 2001. Dynamical spreading of asteroid families via the Yarkovsky effect: the Koronis family and beyond. Science 294, 1693-1696.

Bottke, W.F., Morbidelli, A., Jedicke, R., Petit, J.-M., Levison, H.F., Michel, P., Metcalfe, T.S., 2002. Debiased orbital and absolute magnitude distribution of the near-Earth objects. Icarus 156, 399-433.

Brouwer, D., van Woerkom, A.J.J., 1950. Astronomical Papers Prepared for the Use of the American Ephemeris and Nautical Almanac, vol 13. U.S. Govt. Print. Off., Washington, pp. 81-107.

Brouwer, D., 1951. Secular variations of the orbital elements of minor planets. AJNR 56, 9-32.

Carruba, V., Michtchenko, T.A., Roig, F., Ferraz-Mello, S., Nesvorný, D., 2005. On the Vtype asteroids outside the Vesta family. I. Interplay of nonlinear secular resonances and the Yarkovsky effect: the cases of 956 Elisa and 809 Lundia. A\&A 441, 819-829.

Carruba, V., Michtchenko, T., 2007. Frequency approach to identifying asteroid families. A\&A 475, 1145-1158.

Carruba, V., Michtchenko, T., 2009. A frequency approach to identifying asteroid families. II. Families interacting with nonlinear secular resonances and low-order mean-motion resonances. A\&A 493, 267-282.

Carruba, V., 2009. The (not so) peculiar case of the Padua family. MNRAS 395, 358-377.

Carruba, V., 2010. The stable archipelago in the region of the Pallas and Hansa dynamical families. MNRAS 408, 580-600.

Carruba, V., Morbidelli, A., 2011. On the first $v_{6}$ anti-aligned librating asteroid family of Tina. MNRAS 412, 2040-2051.

Carruba, V., Aljbaae, S., Souami, D., 2014a. Peculiar Euphrosyne. APJ (Acta Pathol. Jpn.) $792,46$.

Carruba, V., Huaman, M., Domingos, R.C., Dos Santos, C.R., Souami, D., 2014 b. Dynamical evolution of V-type asteroids in the central main belt. MNRAS 439, 3168-3179.

Carruba, V., Aljbaae, S., Winter, O.C., 2015. On the Erigone family and the $Z_{2}$ secular resonance. MNRAS 455, 2279-2288.

Carruba, V., Nesvorný, D., Marchi, S., Aljbaae, S., 2016a. Footprints of a possible Ceres asteroid paleo-family. MNRAS 458, 1117-1126.

Carruba, V., Nesvorný, D., 2016. Constraints on the original ejection velocity fields of asteroid families. MNRAS 457, 1332-1338.

Carruba, V., 2016. On the Astrid asteroid family. MNRAS 461, 1605-1613.

Carruba, V., Nesvorný, D., Domingos, R.C., Aljbaae, S., Huaman, M., 2016b. On the highly inclined $\boldsymbol{v}_{W}$ leptokurtic asteroid families. MNRAS 463, 705-711.

Ferraz-Mello, S., 1985. Resonance in regular variables. I - morphogenetic analysis of the orbits in the case of a first-order resonance. CM 35, 209-220.

Ferraz-Mello, S., 2007. Canonical Perturbation Theories, Degenerate Systems and Resonances. Springer, New York.

Gallardo, T., Venturini, J., Roig, F., Gil-Hutton, R., 2011. Origin and sustainability of the population of asteroids captured in the exterior resonance 1:2 with Mars. Icarus 214, 632-644.

Granvik, M., Morbidelli, A., Jedicke, R., Bolin, B., Bottke, W.F., Beshore, E., Vokrouhlický, D., Delbò, M., Michel, P., 2016. Super-catastrophic disruption of asteroids at small perihelion distances. Nature 530, 303-306.

Hirayama, K., 1923. Annales de l'Observatoire Astronomique de Tokyo, p. 11.

Knežević, Z., Milani, A., Farinella, P., Froeschle, C., Froeschle, Ch, 1991. Secular resonances from 2 to $50 \mathrm{AU}$. Icarus 93, 316-330.

Knežević, Z., Milani, A., 2000. Synthetic proper elements for outer main belt asteroids. CM 78, 17-46.

Knežević, Z., Milani, A., 2003. Proper element catalogs and asteroid families. A\&A 403, 1165-1173.

Lemaitre, A., 1994. Hungaria: a potential new family. In: Kozai, Y., Binzel, R.P., Hirayama, T. (Eds.), Seventy-five Years of Hirayama Asteroid Families: the Role of Collisions in the Solar System History, vol 63. Astronomical Society of the Pacific Conference Series, pp. 140-145.

Lemaitre, A., Morbidelli, A., 1994. Proper elements for highly inclined asteroidal orbits. CM 60, 29-56.

Machuca, J.F., Carruba, V., 2012. Secular dynamics and family identification among highly inclined asteroids in the Euphrosyne region. MNRAS 420, 1779-1798.

Milani, A., Knežević, Z., 1990. Secular perturbation theory and computation of asteroid proper elements. CM 49, 347-411.

Milani, A., Knežević, Z., 1992. Asteroid proper elements and secular resonances. Icarus 98, 211-232.

Milani, A., Knežević, Z., 1994. Asteroid proper elements and the dynamical structure of the asteroid main belt. Icarus 107, 219-254.

Milani, A., Knežević, Z., Novaković, B., Cellino, A., 2010. Dynamics of the Hungaria asteroids. Icarus 207, 769-794.

Milani, A., Cellino, A., Knežević, Z., Novaković, B., Spoto, F., Paolicchi, P., 2014. Asteroid families classification: exploiting very large datasets. Icarus $239,46-73$.

Milani, A., Knežević, Z., Spoto, F., Cellino, A., Novaković, B., Tsirvoulis, G., 2017. On the ages of resonant, eroded and fossil asteroid families. Icarus 288, 240-264.

Milić Žitnik, I., Novaković, B., 2015. On some dynamical properties of the Phocaea region. MNRAS 451, 2109-2116.

Morbidelli, A., Henrard, J., 1991. The main secular resonances nu6, nu5 and nu16 in the asteroid belt. CM 51, 169-197.

Morbidelli, A., 1993. Asteroid secular resonant proper elements. Icarus 105, 48-66. 
Nesvorný, D., Brož, M., Carruba, V., 2015. Identification and dynamical properties of asteroid families. In: Michel, P., DeMeo, F.E., Bottke, W. (Eds.), Asteroids IV. University of Arizona Press, pp. 297-321.

Novaković, B., Maurel, C., Tsirvoulis, G., Knežević, Z., 2015. Asteroid secular dynamics: Ceres' fingerprint identified. ApJL 807, L5.

Novaković, B., Tsirvoulis, G., Marò, S., Djošović, V., Maurel, C., 2016. Secular evolution of asteroid families: the role of Ceres. In: Chesley, S., Morbidelli, A., Jedicke, R., Farnocchia, D. (Eds.), The Proceedings of the IAU Symposium 318-Asteroids: New Observations, New Models, pp. 46-54.

Spoto, F., Milani, A., Knežević, Z., 2015. Asteroid family ages. Icarus 257, 275-289.

Tsirvoulis, G., Novaković, B., 2016. Secular resonances with Ceres and Vesta. Icarus 280 300-307.

Vokrouhlický, D., Brož, M., 2002. Interaction of the Yarkovsky-drifting orbits with weak resonances: numerical evidence and challenges. In: Celletti, A., Ferraz-Mello, S., Henrard, J. (Eds.), Modern Celestial Mechanics: from Theory to Applications. Kluwer Academic Publ, Dordrecht, pp. 467-472.

Vokrouhlický, D., Brož, M., Morbidelli, A., Bottke, W.F., Nesvorný, D., Lazzaro, D., Rivkin, A.S., 2006a. Yarkovsky footprints in the Eos family. Icarus 182, 92-117.
Vokrouhlický, D., Brož, M., Bottke, W.F., Nesvorný, D., Morbidelli, A., 2006b. The peculiar case of the Agnia asteroid family. Icarus 183, 349-361.

Vokrouhlický, D., Brož, M., Bottke, W.F., Nesvorný, D., Morbidelli, A., 2006c. Yarkovsky/ YORP chronology of asteroid families. Icarus 182, 118-142.

Vokrouhlický, D., Nesvorný, D., Bottke, W.F., Morbidelli, A., 2010. Collisionally born family about 87 Sylvia. AJNR 139, 2148-2158.

Vokrouhlický, D., Bottke, W.F., Chesley, S.R., Scheeres, D.J., Statler, T.S., 2015. The Yarkovsky and YORP effects. In: Michel, P., DeMeo, F.E., Bottke, W. (Eds.), Asteroids IV. University of Arizona Press, pp. 509-531.

Warner, B.D., Harris, A.W., Vokrouhlický, D., Nesvorný, D., Bottke, W.F., 2009. Analysis of the Hungaria population. Icarus 204, 172-182.

Williams, J.G., 1969. Secular Perturbations in the Solar System. Ph.D. Thesis. University of California, Los Angeles.

Yoshikawa, M., 1987. A simple analytical model for the secular resonance nu6 in the asteroidal belt. Celestial Mech. 40, 233-272.

Zappalà, V., Cellino, A., Farinella, P., Knežević, Z., 1990. Asteroid families. I identification by hierarchical clustering and reliability assessment. AJNR 100, 2030-2046. 